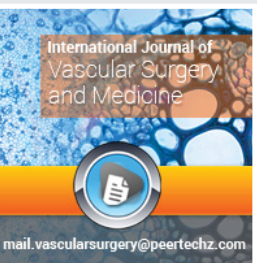

\title{
International Journal of Vascular Surgery and Medicine
}

\section{HS Natraj Setty*, Rahul S Patil, Sumit Bhatnagar, Animesh Guptha, TR Raghu, Jayashree Kharge, BK Geetha, CN Manjunath}

Sri Jayadeva Institute of Cardiovascular Sciences and Research, Bengaluru, Karnataka, India

Dates: Received: 11 January, 2017; Accepted: 15 February, 2017; Published: 16 February, 2017

*Corresponding author: Natraj Setty HS, Doctor, MD, DM, Sri Jayadeva Institute of Cardiovascular Sciences and Research, \#493, 4th Cross, 7th Main, JP Nagar 3rd Phase, Bangalore - 69, Karnataka, India, Tel: 9845612322; 080-26580051; Fax: 080-22977261; E-mail: drnatrajsetty75@gmail.com

Keywords: Rheumatoid arthritis; Acute coronary syndrome; $\mathrm{PCl}$

https://www.peertechz.com

\section{Case Report}

Rheumatoid Arthritis and Acute Coronary Syndrome: Changing Clinical Presentation

\section{Abstract}

Rheumatoid arthritis is a chronic systemic inflammatory disease, Rheumatoid arthritis can affect the pericardium, myocardium, and endocardium. RA increases cardiovascular morbidity and mortality by accelerated atherosclerosis. Patients with RA are at a twofold increased risk for myocardial infarction and stroke. Rheumatoid arthritis is often complicated by thrombocytosis. Thrombocytosis usually occurs during the active clinical stages of RA. We report a 40 year old male who presented with sudden onset of back pain, neck pain and gum bleeding he was diagnosed to have Acute Anterior Wall Myocardial Infarction (AWMI) and successfully under went $P C I$ to $L A D$ with uneventful follow up.

\section{Background}

Atherosclerosis has been traditionally considered a lipiddriven disorder affecting the arterial system. Autoimmune diseases, a group of disorders arising from abnormal autoimmunity, can involve different organ systems with a wide spectrum of clinical manifestations. Coronary revascularization with percutaneous coronary intervention (PCI), the most frequently used revascularisation procedure for Coronary Artery Disease in autoimmune diseases. Controlling cardiovascular risk factors, reducing the cardiovascular disease burden in Rheumatoid arthritis requires early detection of symptoms and prompt intervention. We report a 40-year-old male presented with sudden onset of back pain, neck pain and gum bleeding he successfully underwent primary PCI to LAD.

\section{Case Profile}

A 40-year-old male was admitted with complaints of neck pain, back pain and gum bleeding sudden in onset. His ECG was showing ST segment elevation myocardial infarction (Figure 1. AWMI). Routine blood investigations were normal, other investigations showed elevated acute phase reactants, elevated RA factor and troponins. He had history of RA for ten years and was on corticosteroids and Non-Steroidal Anti Inflammatory Drugs (NSAID's). The Patient had no other associated risk factors and no family history of coronary artery disease. Echocardiography revealed regional wall motion abnormality Ejection fraction (EF) $30 \%$. The Patient was taken for cardiac catheterization, Coronary angiography revealed proximal LAD has total occlusion. Successful PCI was done to LAD using drug eluting stent (DES) 3X24mm deployed at 12 ATM (Figure 2A,2B showing angiographic finding in LAD before and after PCI). patient showed prompt recovery and remained asymptomatic on follow up.

\section{Discussion}

Rheumatoid arthritis is a chronic systemic inflammatory disease RA can affect the pericardium, myocardium, and endocardium [1]. Moreover, RA increases cardiovascular morbidity and mortality by accelerated atherosclerosis. Cardiovascular mortality accounts for $40-50 \%$ of all deaths in RA. Patients with RA are at a twofold increased risk of myocardial infarction and stroke [2]. The increased

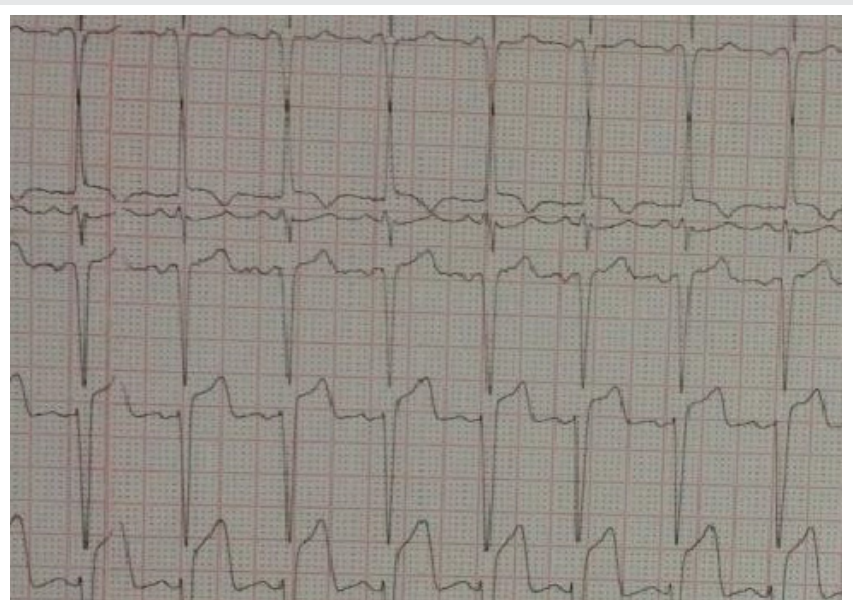

Figure 1: Electro Cardiogram showing ST segment elevation in anterior chest leads. 

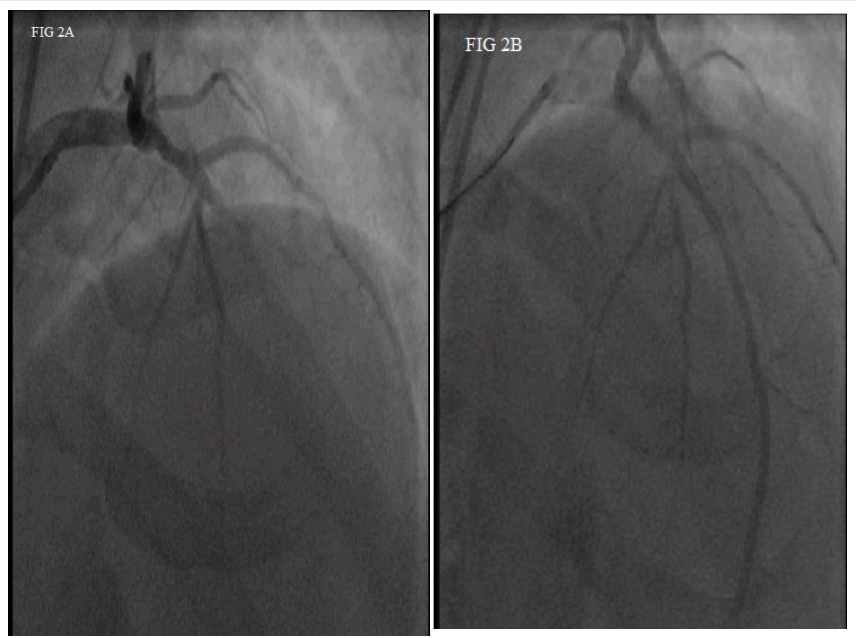

Figure 2: A) Coronary Angiography showing mid LAD has total occlusion. B) Coronary Angiography showing after $\mathrm{PCl}$ with TIMI 3 flow.

cardiovascular disease risk in RA patients is independent of cardiovascular risk factors. Pathogenic mechanisms include pro-oxidative dyslipidemia, insulin resistance, prothrombotic state, hyperhomocysteinemia, and immune mechanisms such as $\mathrm{T}$-cell activation that subsequently leads to endothelial dysfunction and arterial stiffness, which are the congeners of accelerated atherosclerosis observed in RA patients [3]. Markers of systemic inflammation (eg, interleukin [IL]-6, IL-17, tumor necrosis factor [TNF]) have been correlated with increased the risk of cardiovascular death in patients with rheumatoid arthritis [4]. The high burden of cardiovascular diseases in patients with autoimmune diseases has drawn attention to whether autoimmune diseases might worsen the prognosis for Coronary Artery Diseases. Outcomes after acute coronary syndrome have been compared in patients with autoimmune diseases, such as rheumatoid arthritis and Systemic lupus erythematosus [5], although some patients undergo coronary revascularization with percutaneous coronary intervention. In the modern era of widespread use of intervention therapy, the outcomes of PCI in patients with autoimmune diseases remain largely underdetermined. The impact of autoimmune diseases on patients undergoing PCI requires further elucidation [6].

\section{Conclusion}

Patients with Rheumatoid arthritis are at increased risk for the development of Coronary Artery Disease and associated morbidity and mortality. Cardiovascular disease often goes unrecognized in patients with Rheumatoid arthritis therefore it is prudent to carefully assess all patients with Rheumatoid arthritis. Substantial evidence suggests that chronic systemic inflammation contributes significantly to excess cardiovascular disease in Rheumatoid arthritis and that effective suppression of Rheumatoid arthritis -associated inflammation appears to reduce mortality. Classical cardiovascular risk factors play a role and may, in some cases, be exacerbated by the medications used to treat Rheumatoid arthritis. Prevention of cardiovascular disease in Rheumatoid arthritis requires a combined approach incorporating cardiovascular risk-factor screening, management and Coronary Interventions and most importantly effective and sustained control of Rheumatoid arthritis disease activity.

\section{References}

1. Lebowitz WB (1963) The heart in rheumatoid arthritis (rheumatoid disease). A clinical and pathological study of sixty-two cases. Ann Intern Med 58: 102-123. Link: https://goo.gl/EE003d

2. Knonkaert DC (2007) Cardiac involvement in systemic inflammatory diseases Eur Heart J 28: 1797-1804. Link: https://goo.gl/uoqz6f

3. del Rincón ID1, Williams K, Stern MP, Freeman GL, Escalante A (2001) High incidence of cardiovascular events in a rheumatoid arthritis cohort not explained by traditional cardiac risk factors. Arthritis Rheum 44: 2737-2745. Link: https://goo.gl/myRliu

4. Sattar N, McCarey DW, Capell H, Mclnnes IB (2003) Explaining how "highgrade" systemic inflammation accelerates vascular risk in rheumatoid arthritis. Circulation 108: 2957-2963. Link: https://goo.gl/wKGVOB

5. Ward MM (2004) Outcomes of hospitalizations for myocardial infarctions and cerebrovascular accidents in patients with systemic lupus erythematosus. Arthritis Rheum 50: 3170-3176. Link: https://goo.gl/BbX5nk

6. Maksimowicz-McKinnon K, Selzer F, Manzi S, Kip KE, Mulukutla SR, et al (2008) Poor 1-year outcomes after percutaneous coronary interventions in systemic lupus erythematosus: report from the National Heart, Lung, and Blood Institute Dynamic Registry. Circ Cardiovascular Interv 1: 201-208. Link: https://goo.gl/7lkcu7

Copyright: @ 2017 Natraj Setty HS, et al. This is an open-access article distributed under the terms of the Creative Commons Attribution License, which permits unrestricted use, distribution, and reproduction in any medium, provided the original author and source are credited. 